\title{
Impact of organized intraseasonal convective perturbations on the tropical circulation
}

\author{
Hugo Bellenger, ${ }^{1}$ Jean-Philippe Duvel, ${ }^{1}$ Matthieu Lengaigne, ${ }^{2}$ and Phu Levan ${ }^{1}$ \\ Received 18 June 2009; revised 17 July 2009; accepted 22 July 2009; published 25 August 2009.
}

[1] Using an atmospheric Global Circulation Model (GCM), we study the dynamical response of the tropical atmosphere to large-scale convective perturbations. We hypothesize that such perturbations can be triggered by intraseasonal SST anomalies. We thus compare two ensembles of simulations forced either with actual daily SST fields (high frequency, $\mathrm{HF}$ ) or with low-pass filtered SST fields ( $\mathrm{T}>90$ days; low frequency, LF). In HF simulations, there is a small increase of seasonal mean precipitation, but a strong and statistically significant increase of the low-level westerly flow. It is argued that it is mostly the "aggregation" of the convection into a few large-scale organized intraseasonal events, and not the intensification of the seasonal mean precipitation, that acts to intensify the low-level westerly flow in the HF simulations. This non-linear behavior shows that a correct representation of intraseasonal variability is required for a correct simulation of the average tropical circulation. Citation: Bellenger, H., J.-P. Duvel, M. Lengaigne, and P. Levan (2009), Impact of organized intraseasonal convective perturbations on the tropical circulation, Geophys. Res. Lett., 36, L16703, doi:10.1029/2009GL039584.

\section{Introduction}

[2] The tropical intraseasonal variability (ISV; 20-60 day) is characterized by large-scale convective and dynamical perturbations in the Indo-Pacific region. It is a very important mode of variability in the tropics that modulates in particular the Asian summer monsoon. However, global circulation models (GCMs) generally have difficulties in simulating these large-scale perturbations. The simulations are generally improved by coupling the atmospheric GCM to a full or a slab ocean model [Fu and Wang, 2004], but these coupled GCMs also have difficulties to reproduce the observed ISV. Compared to observation, these models generally simulate convective perturbations less spatially organized and with a perturbation pattern less reproducible from one intraseasonal event to another [Xavier et al., 2008]. Such a relatively dispersed convective heating in the model gives a weak Matsuno-Gill-type [Gill, 1980] dynamical response. This can prevent the model to simulate a realistic ISV and this can also impact the average seasonal circulation in the model [Bellenger and Duvel, 2007].

[3] In situ and satellite measurements show that intraseasonal events in the Indo-Pacific region are associated to SST perturbations that can reach a few degrees [Harrison and

\footnotetext{
${ }^{1}$ Laboratoire de Météorologie Dynamique, Ecole Normale Superieure, Paris, France.

${ }^{2}$ Laboratoire d'Océanographie et de Climatologie : Expérimentations et Analyses, CNRS, Paris, France.
}

Copyright 2009 by the American Geophysical Union. 0094-8276/09/2009GL039584
Vecchi, 2001; Duvel et al., 2004]. It is thus interesting to analyze the ISV response of a GCM to intraseasonal SST anomalies. However, previous studies using realistic or idealized intraseasonal SST perturbations [Matthews, 2004; Fu and Wang, 2004] noted that the forcing approach might be problematic. The SST evolution, prescribed from observations, is indeed not necessarily consistent with the evolution of the surface fluxes given by the GCM. Fu and Wang [2004] showed that the physical parameterization of the model tends to give an in-phase relationship between the convection and the SST instead of the quadrature that is generally observed [e.g., Duvel and Vialard, 2007]. For these reasons, the forced approach is not adapted to fully represent the physical mechanisms explaining the ISV. However, the forced approach can still give interesting results for process studies. In particular, since the simulated convection is sensitive to the SST in GCMs, large-scale intraseasonal SST anomalies should trigger large-scale organized intraseasonal convective perturbations. The convective heating associated to these slowly-evolving intraseasonal SST perturbations may be considered as stationary relative to the Matsuno-Gill dynamical response that sets up. Using such simulations, it is thus theoretically possible to analyze the impact of this organized convective ISV on the large-scale atmospheric response. This is the main objective of the present study.

[4] This study is based on two ensembles of atmospheric simulations forced either with the actual daily SST or with SST filtered at low frequency (periods larger than 90 days) in which intraseasonal SST anomalies are reduced. This approach conserves the seasonal average SST and thus gives similar average boundary conditions. The actual daily SST fields are given by the Tropical Rainfall Measuring Mission's (TRMM) Microwave Imager (TMI) [Wentz et al., 2000]. TMI can estimate the SST in cloudy conditions and gives intraseasonal SST variability larger by a factor of two compared to other satellite observations [Duvel and Vialard, 2007]. This study focuses on the Indian Ocean where strong and recurrent intraseasonal variations of the SST are observed south of the equator from December to March. We will not comment results for the Pacific Ocean since intraseasonal SST perturbations are weaker here [Duvel and Vialard, 2007] and have a smaller impact on the convection. The ability of the model to simulate such organized and reproducible ISV of convection and wind is diagnosed using the Local Mode Analysis (LMA) [Goulet and Duvel, 2000; Duvel and Vialard, 2007].

[5] Section 2 describes the GCM experiments and the analysis approach. In section 3, we verify that intraseasonal perturbations of surface wind and surface flux are enhanced in the HF simulations and we look for differences in the seasonal averages. Section 4 contains a more detailed analysis of the spatial organization of the intraseasonal perturbations. In section 5, we show that the simulated 


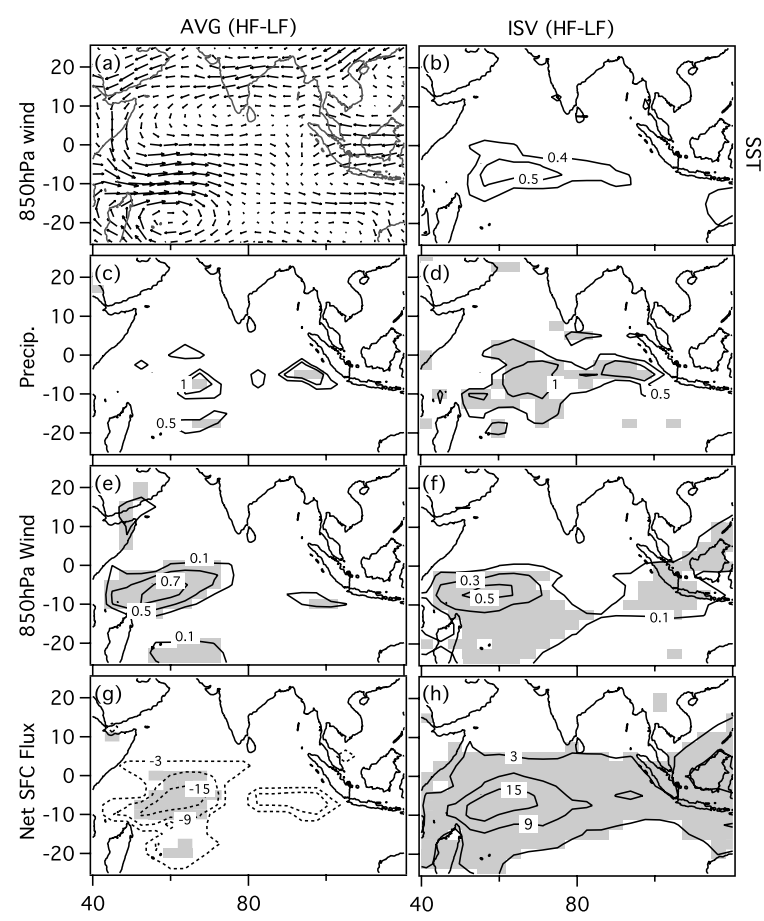

Figure 1. Seasonal December-March (DJFM) average of (a) difference between HF and LF of $850 \mathrm{hPa}$ wind average (AVG, vector of $10^{\circ}$ length is $1 \mathrm{~ms}^{-1}$ ) and (b) TMI SST 20-60 day standard deviation (ISV) (K) for 1998-2003; and the DJFM average difference (HF-LF) of AVG and ISV for: (c and $\mathrm{d}$ ) the precipitation ( $\mathrm{mm} /$ day); (e and $\mathrm{f}$ ) the $850 \mathrm{hPa}$ wind speed $\left(\mathrm{ms}^{-1}\right)$; and $(\mathrm{g}$ and $\mathrm{h})$ the net surface flux (positive downward, $\mathrm{Wm}^{-2}$ ). Negative values are dashed. The differences shown are significant at $95 \%$ by a student t-test on the 5 times 9 winter ensembles (HF and LF), the $99 \%$ level is shaded.

large-scale dynamical response is also more realistic and reproducible in $\mathrm{HF}$.

\section{AGCM Experimental Setup and Analysis Approaches}

[6] Two ensemble simulations are performed using the LMDZ-4 AGCM with a regular horizontal resolution of $3.75^{\circ}$ longitude $\times 2.5^{\circ}$ latitude and with 19 levels in the vertical. A detailed description of the LMDZ-4 model and its performance is given by Hourdin et al. [2006]. Two ensembles of 9 integrations are performed for the 19982003 period. The model is forced using daily TMI SST for latitude lower than $35^{\circ}$, a blending of TMI and Reynolds SST [Reynolds and Smith, 1994] between 35 and $40^{\circ}$ and only Reynolds SST for higher latitudes. A High Frequency (HF) ensemble is forced using this time series and a Low Frequency (LF) ensemble is forced with a filtered version of this time series obtained by removing the period lower than 90 days (only harmonics 1 to 24 are conserved for the 2191-day time series between January 1998 and December 2003). The SST forcing is the same for each member of a given ensemble and initial atmospheric, continental surface and snow cover conditions are taken from ERA 40 in the beginning of December 1997 with a one-day lag between the different members.
[7] The Local Mode Analysis (LMA) [Goulet and Duvel, 2000; Duvel et al., 2004; Duvel and Vialard, 2007] is used to extract the main characteristics of convective and wind intraseasonal perturbations. The LMA is based on a series of Complex EOFs (CEOFs) computations performed for relatively small time sections (120 days) running along a time series (every 5 days). Maxima in the time series of the first-CEOF variance-percentage give the collection of time sections centered on perturbations, or events, that are wellorganized at large scale. Applied to the precipitation time series in the intraseasonal spectral band (harmonics 1 to 6 , i.e., $20 \leq \mathrm{T} \leq 120$ days), the LMA identifies the main intraseasonal convective events and gives their corresponding spatial patterns. Each event is also associated to a normalized spectrum (or spectral key) that contains the temporal characteristics of the large-scale organized intraseasonal event and that may be used to compute the corresponding spatial patterns (spatial distribution of amplitude and relative phase) of different parameters [Duvel and Vialard, 2007]. This gives, for each intraseasonal event, the surface wind pattern associated to the convective perturbations pattern. Using such an approach, it is also possible to compute average patterns and the degree of resemblance between these average patterns and the pattern of each intraseasonal event. This degree of resemblance is given by a normalized distance metric, and the distribution of these distances makes it possible to quantify the reproducibility of the pattern of the studied phenomenon. In our case this gives in particular the reproducibility of the simulated atmospheric response to intraseasonal SST forcing. The LMA is performed here for LF, HF, Global Precipitation Climatology Project (GPCP) daily precipitation [Huffman et al., 2001] and NCEP $850 \mathrm{hPa}$ wind. Prior to LMA, we remove seasonal and interannual variations by filtering out periods larger than 120 days for both model and observation.

[8] For a given region and a given intraseasonal event, the LMA variance is thus the part of the signal having common spectral characteristics with other regions (the spectral key). The ratio, noted $R_{0}$, between the LMA variance and the variance of the 20-90 day signal for the corresponding time section is thus an indicator of the part of the local signal corresponding to the large-scale organized intraseasonal perturbation. The map of the ratio $R_{o}$ averaged over all intraseasonal events thus highlights the distribution of the impact of large-scale organized events on the local intraseasonal variability. If the simulated intraseasonal variability is not well spatially organized, $R_{o}$ will be small for most regions compared to observations.

\section{Intraseasonal Variance and Seasonal Average}

[9] As shown in previous studies, the intraseasonal SST variability in the west and central Indian Ocean is maximal south of the equator during boreal winter (Figure 1b). The sensitivity of the AGCM to this intraseasonal SST forcing is quantified by the difference between ensemble averages for $\mathrm{HF}$ and LF. We examine first intraseasonal (20-60 day band) standard deviation maps for precipitation, surface zonal wind and net surface flux. For the precipitation (Figure 1d), the HF ISV is reinforced over west and central Indian Ocean in direct association to the local SST forcing. This is mostly due to the strong sensitivity of the GCM 


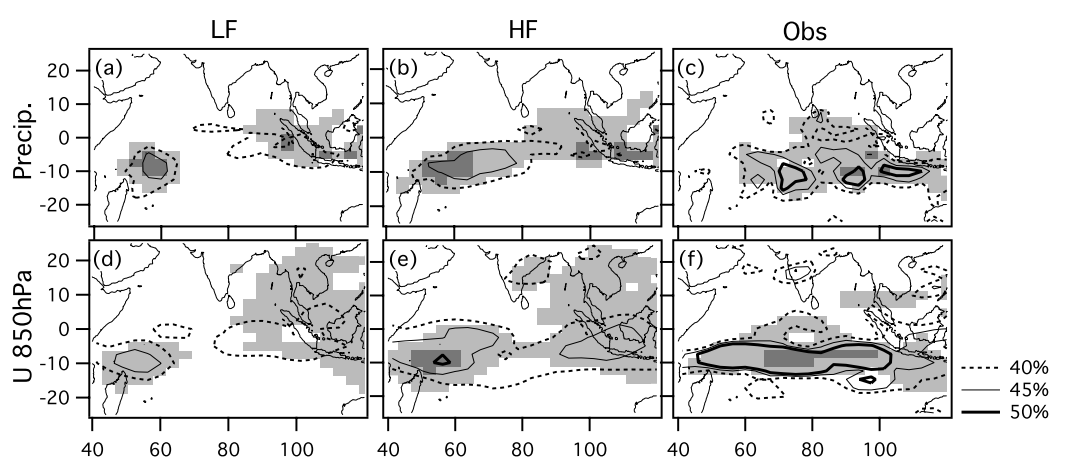

Figure 2. Average LMA ISV amplitude (shaded) and average ratio $R_{o}$ (contours) of $(a-c)$ precipitation perturbations and (b-f) $850 \mathrm{hPa}$ zonal wind perturbations from December to March for LF and HF ensembles and the observations. The grey levels are $2.5 \mathrm{~mm} /$ day light grey and $4 \mathrm{~mm} /$ day dark grey for precipitation and $1.5 \mathrm{~ms}^{-1}$ light grey and $2.5 \mathrm{~ms}^{-1}$ dark grey for the $850 \mathrm{hPa}$ zonal wind.

convective parameterization to the observed intraseasonal variations of the SST. The associated ISV of the surface wind (Figure 1f) is reinforced to the west and is consistent with a Gill-type [Gill, 1980] dynamical response that gives a maximum westerly (easterly) wind perturbation west (east) of the tropospheric warm anomaly. The surface fluxes ISV (Figure 1h) is also reinforced in HF over the western Indian Ocean, due to the reinforced ISV for both surface wind and precipitation (i.e., convective cloudiness reducing the surface solar flux).

[10] The reinforcement of the ISV of atmospheric parameters in HF produces non-linear effects modifying the seasonal mean values. These modifications are generally not significant at the $99 \%$ level for the precipitation (Figure 1c) but are relatively large and significant for the $850 \mathrm{hPa}$ wind speed (Figure 1e) and the net surface flux (Figure 1g). In $\mathrm{HF}$, the $850 \mathrm{hPa}$ seasonal average wind speed is increased by up to $20 \%$ in the western Indian Ocean. The reinforcement of the convective ISV in HF thus affects the seasonal mean surface wind and heat fluxes.

[11] The increase of the seasonal average low-level wind corresponds to an intensification of the westerlies to the west of the convection (Figures 1a, 1c, and 1d). This may not be only a response to an enhanced convective activity as there is only a small variation of the mean precipitation (Figure 1c). This enhanced dynamical perturbation could be rather mostly due to non-linear effect related to the better large-scale organization of the convective event in HF. This point is addressed in the following section.

\section{Spatial Organization of the Convective Perturbations}

[12] Figure 2 shows the average LMA amplitude and the average ratio $R_{o}$ for LF and HF simulations and for observations. This is a diagnostic of the spatial organization of simulated intraseasonal convective perturbations (precipitation) and of the corresponding low-level wind response ( $850 \mathrm{hPa}$ zonal wind). We only report the zonal wind perturbations since Gill-type response to convective heating is mostly zonal near the equator. The interpretation is thus easier. Moreover change in the average wind speed (Figure 1e) is also mostly due to the zonal component (Figures 1a-1e).
[13] The observed ISV of the precipitation (Figure 2c) is stronger and better organized (large $\mathrm{R}_{\mathrm{o}}$ ) south of the equator and between 70 and $100^{\circ} \mathrm{E}$. In agreement with a Gill-type response, the maximum zonal low-level wind ISV (Figure 2f) is observed west of the main convective ISV centers. In LF simulations, precipitation (Figure 2a) and wind (Figure 2d) ISV are weak and poorly organized compared to observations. This shows that the GCM forced with slowly evolving SST has a poor skill in producing organized ISV of the convection above this region. In particular, the ISV of the zonal wind is very small and limited to the southwest Indian Ocean. The $\mathrm{R}_{\mathrm{o}}$ factor for the wind is not clearly located west of the convective perturbation, suggesting that the organized Gill-type response is not very efficient in LF. The role of the intraseasonal SST forcing in organizing the convective perturbation in the GCM is evident when comparing LF and HF ensembles (Figures 2a and $2 b$ ). The precipitation ISV is stronger and better organized south of the Equator for the $\mathrm{HF}$ ensemble. The main convective ISV center, around $60^{\circ} \mathrm{E}$, is shifted to the west in HF compared to observations, in clear relation with the location of the maximum ISV of the SST (Figure 1b). This is an expected drawback of the forcing of a GCM with observed SST. As shown by Duvel and Vialard [2007] the largest intraseasonal SST perturbation is indeed located to the west of the convective perturbation, mostly in relation to the strong westerly wind perturbation associated to the Gill-type response. Here, the GCM convection is sensitive to these SST perturbations, resulting in a convective ISV center shifted to the west. Interestingly, and despite this expected drawback, a large and organized low-level wind response appears in the HF ensemble (Figure 2e). This wind response is well located to west of the convective ISV center (Figure 2b), suggesting that a Gill-type response is at work in the HF ensemble.

\section{Reproducibility and Realism of the Pattern}

[14] As discussed in section 2, the LMA also offers the possibility to test the reproducibility and the realism of the pattern of the different intraseasonal events. For observation, and for each ensemble, the reproducibility is estimated by computing the normalized distance between the pattern of each event and the corresponding seasonal average pattern. If this distance is small for most events, the average pattern well represents them and the phenomenon is well 


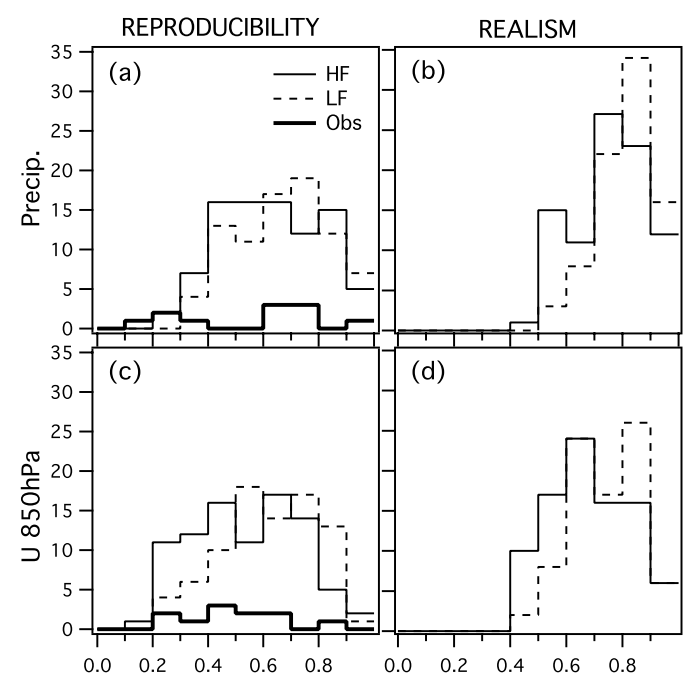

Figure 3. Distributions of distances between the pattern of each intraseasonal event and either (a and c) the corresponding average DJFM mode (Reproducibility), or (b and d) the observed average DJFM mode (Realism). The distance distributions are shown for precipitation patterns (Figures 3a and $3 \mathrm{~b}$ ) and for $850 \mathrm{hPa}$ zonal wind patterns (Figures $3 \mathrm{c}$ and $3 \mathrm{~d}$ ), and for LF (dashed), HF (solid thin) and observations (solid thick).

reproducible. In observation, the average winter pattern well represents the pattern for 4 intraseasonal events out of 11 for the precipitation (distance less than 0.4, Figure 3a). The reproducibility is also good for the associated average wind pattern with 6 modes out of 11 with a distance less than 0.5 (Figure 3c). For the GCM simulations (Figures 3a and 3c), the precipitation patterns are slightly more reproducible in HF than in LF (Figure 3a). This is due mostly to the reproducible nature of the observed SST ISV and to its role in organizing the convection in HF. More interestingly, the wind response (Figure 3c) is also more reproducible. The degree of realism of the simulated ISV patterns may be also assessed by computing the normalized distance between the pattern of each simulated event and the observed seasonal average pattern. Since the observed reproducibility is good (Figures 3a and 3c), a small distance will indicate that the simulated intraseasonal event has a realistic pattern. Realism of the convective perturbations is slightly better for HF than for LF (Figure 3b). Moreover, the associated wind response pattern is also more realistic for HF than for LF (Figure 3d). The SST forced organization of the convection in HF thus leads to a more realistic representation of the low-level wind ISV, and this despite the westward shift of the main convective ISV center in regard to observations.

\section{Discussion}

[15] While the seasonal average SST is not changed, the precipitation increases slightly over the central Indian Ocean in the HF simulations compared to LF. However, this result could depend mostly on the convective parameterization of the LMD GCM that tends to increase the convection over warm SST. In addition, in contrast with the wind difference between HF and LF, the seasonal average precipitation difference is not significant at the
$99 \%$ level. The origin of this slight non-linear effect is thus questionable and deserves further study. The main result of this study is that the intraseasonal SST variability increased the large-scale organization, the reproducibility and the realism of the precipitation pattern of the different intraseasonal events simulated by the GCM. The associated lowlevel wind intraseasonal perturbation patterns are also more organized (Figure 2e), more reproducible and more realistic in $\mathrm{HF}$, and this results in a larger average low-level westerly flow over the western Indian Ocean (Figure 1e). It is deduced that, in HF simulations, the aggregation of the convection into a few large-scale organized events gives a non-linear dynamical response that impacts the average circulation. In LF simulations, the convection is more disseminated in both space and time and does not generate such an organized large-scale dynamical response.

[16] The importance of these non-linear effects cannot be fully addressed from forced atmospheric GCM simulations. However, as shown by Xavier et al. [2008], intraseasonal SST perturbations are usually weak in current coupled GCMs compared to observations. This is partly due to the coarse vertical resolution of the Ocean model near the surface and to the absence of processes such as the formation of diurnal warm layers in calm conditions [see, e.g., Bernie et al., 2005; Bellenger and Duvel, 2009]. As demonstrated by the wrong phase relationship between SST and convective perturbations in forced simulations (see the Introduction), this could also be due to the convective schemes that respond too rapidly to surface warming and prevent the development of large positive SST anomalies. In a coupled system, it can be hypothesized that the processes at the origin of the triggering of a large-scale convective perturbation may differ from the coupling processes (surface, convection and dynamics) explaining the propagation of the intraseasonal perturbation. We showed here that an intraseasonal SST perturbation is one possible process to produce a large-scale organized convective perturbation that may latter evolve and propagate as an intraseasonal perturbation. This propagation (eastward for the MJO, northward for intraseasonal perturbation during summer) of the coupled intraseasonal perturbation is related to different coupled processes that have been already proposed in the literature [see, e.g., Zhang, 2005]. To conclude, it appears that the triggering of large-scale organized convective perturbation could be a necessary process to correctly simulate the intraseasonal variability and the tropical largescale circulation in GCMs.

[17] Acknowledgments. TMI data are produced by Remote Sensing Systems and sponsored by the NASA Earth Science MEaSUREs DISCOVER Project. Data are available at www.rmss.com. H. Bellenger was funded by the Centre National d'Etudes Spatiales (CNES). We thank the two anonymous referees for their very useful comments.

\section{References}

Bellenger, H., and J. P. Duvel (2007), Intraseasonal perturbations related to the seasonal march of the Indo-Pacific monsoons, J. Clim., 20, 2853-2863, doi:10.1175/JCLI4182.1.

Bellenger, H., and J. P. Duvel (2009), An analysis of tropical ocean diurnal warm layers, J. Clim., 22, 3629-3646.

Bernie, D. J., S. J. Woolnough, J. M. Slingo, and E. Guilyardi (2005), Modeling diurnal and intraseasonal variability of the ocean mixed layer, J. Clim., 18, 1190-1202, doi:10.1175/JCLI3319.1.

Duvel, J. P., and J. Vialard (2007), Indo-Pacific sea surface temperature perturbations associated with intraseasonal oscillation of the tropical convection, J. Clim., 20, 3056-3082, doi:10.1175/JCLI4144.1. 
Duvel, J. P., R. Roca, and J. Vialard (2004), Ocean mixed layer temperature variations induced by intraseasonal convective perturbations over the Indian Ocean, J. Atmos. Sci., 61, 1004-1023, doi:10.1175/1520-0469(2004)061< 1004:OMLTVI>2.0.CO;2.

Fu, X., and B. Wang (2004), Differences of boreal summer intraseasonal oscillations simulated in an atmosphere-ocean coupled model and an atmosphere-only model, J. Clim., 17, 1263-1271, doi:10.1175/1520-0442 (2004)017<1263:DOBSIO >2.0.CO;2.

Gill, A. E. (1980), Some simple solutions for heat-induced tropical circulation, Q. J. R. Meteorol. Soc., 106, 447-462, doi:10.1002/qj.49710644905.

Goulet, L., and J. P. Duvel (2000), A new approach to detect and characterize intermittent atmospheric oscillations: Application to the intraseasonal oscillation, J. Atmos. Sci., 57, 2397-2416, doi:10.1175/1520-0469(2000) 057<2397:ANATDA $>2.0 . \mathrm{CO} ; 2$.

Harrison, D. E., and G. A. Vecchi (2001), January 1999 Indian Ocean cooling event, Geophys. Res. Lett., 28, 3717-3720, doi:10.1029/2001GL013506.

Hourdin, F., et al. (2006), The LMDZ4 general circulation model: Climate performance and sensitivity to parametrized physics with emphasis on tropical convection, Clim. Dyn., 27, 787-813, doi:10.1007/s00382-006-0158-0.

Huffman, G. J., R. F. Adler, M. Morrissey, D. T. Bolvin, S. Curtis, R. Joyce, B. McGavock, and J. Susskind (2001), Global precipitation at one-degree daily resolution from multi-satellite observations, J. Hydrometeorol., 2 36-50, doi:10.1175/1525-7541(2001)002<0036:GPAODD>2.0.CO;2.
Matthews, A. J. (2004), The atmospheric response to observed intraseasonal tropical sea surface temperature anomalies, Geophys. Res. Lett., 31, L14107, doi:10.1029/2004GL020474.

Reynolds, R. W., and T. M. Smith (1994), Improved global sea surface temperature analyses using optimum interpolation, J. Clim., 7, 1195-1202, doi:10.1175/1520-0442(1994)007<0929:IGSSTA > 2.0.CO;2

Wentz, F. J., C. Gentemann, D. Smith, and D. Chelton (2000), Satellite measurements of sea-surface temperature through clouds, Science, 288, 847-850, doi:10.1126/science.288.5467.847.

Xavier, P. K., J. P. Duvel, and F. J. Doblas-Reyes (2008), Boreal summer intraseasonal variability in coupled seasonal hindcasts, J. Clim., 21, 4477-4497, doi:10.1175/2008JCLI2216.1.

Zhang, C. (2005), Madden-Julian Oscillation, Rev. Geophys., 43, RG2003, doi:10.1029/2004RG000158.

H. Bellenger, J.-P. Duvel, and P. Levan, Laboratoire de Météorologie Dynamique, Ecole Normale Superieure, 24 rue Lhomond, F-75231 Paris CEDEX, France. (belleng@1md.ens.fr)

M. Lengaigne, Laboratoire d'Océanographie et de Climatologie : Expérimentations et Analyses, CNRS, Boîte 100, 4, place Jussieu, F-75252 Paris CEDEX 05, France. 